\title{
Integrated Roll-Pitch-Yaw Autopilot Design for Missiles
}

\author{
Yoon-Hwan Kim*, Dae-Yeon Won*, Tae-Hun Kim*, Min-Jea Tahk** \\ Department of Aerospace Engineering \\ Korea Advanced Institute of Science and Technology, \\ Daejeon, Korea
}

\author{
Byung Eul Jun***, Jin-Ik Lee***, and Jo Young An*** \\ Agency for Defense Development \\ Daejeon, Korea
}

\begin{abstract}
An roll-pitch-yaw integrated autopilot for missiles is designed for compensation of dynamics coupling. The proposed autopilot is based on the classical control technique. The gains of the proposed autopilot are optimized by using co-evolutionary augmented Lagrangian method(CEALM). Several cost functions are compared in order to find feasible control gains. For a case that a bank angle of missiles is unknown, multiple models are used in the autopilot optimization. In nonlinear simulations as well as linear simulations, the proposed autopilot provided good performances.
\end{abstract}

Key Words : Autopilot design, Dynamics coupling, Missile, Parameter optimization

\section{Introduction}

Recent improvements in aircraft and missile systems have expanded the flight envelope. In most cases, the missile autopilot design has been achieved in roll, pitch and yaw channel, respectively. However, since the missile has highly coupled dynamics in high angle of attack, the existing autopilot design techniques in separate channels cannot cover whole flight envelope.

The missile dynamic properties has the cross coupling in roll, pitch and yaw channel, and the effect of the cross coupling changed during flight can be large enough to break the stability of the control system. The kinematic cross coupling and the inertial cross coupling are predictable, but it is difficult to know induced roll produced by aerodynamic uncertainties precisely. In the case of cruciform missile configuration, unsymmetric flow patterns arise when the angle of attack and the sideslip angle are not identical. Then the induced roll moment is produced even if the control input is zero by unsymmetric flow patterns on the fins. Besides, because of the small moment of inertia on the roll channel, the induced roll moment can cause the fatal performance degradations. The induced roll moment is highly nonlinear and affected by the angle of attack, Mach No. and configuration of missile [1, 2].

This paper describes the roll-pitch-yaw integrated autopilot design based on the 5-DOF missile model by considering the dynamics coupling. Although the autopilot can be designed in roll, pitch and yaw channel, separately, this existing approach could not compensate cross coupling effects [3]. Thus we design the roll-pitch-yaw integrated autopilot for the highly maneuverable missile by considering the induced roll moments. Based on 5-DOF missile models, the integrated autopilot design problem is the problem of dealing with multi-input multi-output(MIMO) systems.

* Ph. D. Candidate

** Professor

E-mail : mjtahk@fdcl.kaist.ac.kr Tel : 042-869-3718 $\quad$ Fax : 042-869-3710

*** Researcher 
This problem cannot be solved with the classical control techniques. Therefore, we compose fixed autopilot structures and perform parameter optimizations in order to find optimal gain set. Parameter optimization for aircraft control design has been proposed in Ref. [4, 5]. The authors state that parameter optimization techniques are useful to find control gains which minimize the performance index designed by user. In this paper, $\mathrm{co}^{-}$evolutionary augmented Lagrangian method (CEALM) [6] is applied to design optimal gains with fixed autopilot structures. As an integrated autopilot structure, we considered two approaches using cross-feed loops between a pitch and a roll channel as well as a yaw and a roll channel.

This paper is organized as follows: in the next section, the 5-DOF missile dynamic model is presented. The third section illustrates integrated autopilot structures and several cost functions for the optimization of autopilot gains. In the fourth section, the performances of the proposed integrated autopilot are analyzed on single and multiple linear model which are linearized at different bank angles. The final section summarizes the overall results.

\section{Missile Model}

\section{5-DOF Missile Model}

In this paper, the approach of autopilot design assumes that the missile is modeled by a 5-DOF model. The 5-DOF model is modeled by 6 -DOF model with the fixed altitude and longitudinal velocity. Also, it is assumed that the missile is a rigid body and has the symmetric cruciform missile configuration. Under these assumptions, the 5-DOF rigid-body equations of motions are expressed by the differential equations describing the translational motion and rotational motion as follows.

$$
\begin{aligned}
& \dot{v}=-r u_{0}+p w+F_{y} / m \\
& \dot{w}=-p v+q u_{0}+F_{z} / m \\
& \dot{p}=L / I_{x x} \\
& (1) \dot{q}=-\left(I_{x x}-I_{z z}\right) / I_{y y} p r+M / I_{y y} \\
& \dot{r}=-\left(I_{y y}-I_{x x}\right) / I_{z z} p q+N / I_{z z}
\end{aligned}
$$

where $(u, v, w)$ are the longitudinal, lateral and vertical body velocities, $(p, q, r)$ are the roll, pitch and yaw body rates, $\left(I_{x x}, I_{y y}, I_{z z}\right)$ is the inertial moments, $\left(F_{y}, F_{z}\right)$ are the external forces and $(L, M, N)$ are the external moments. For the missile, the external forces and moments are produced by aerodynamics forces and nonlinear functions of angle of attack, bank angle and deflection angles.

\section{Actuator Model}

The actuator is modeled by a second-order transfer function between the commanded fin deflection and the actual fin deflection as Eq. (2).

$$
\frac{\delta}{\delta_{c m d}}=\frac{\omega_{n}^{2}}{s^{2}+2 \zeta \omega_{n} s+\omega_{n}^{2}}
$$

where $\delta$ is the actual fin deflection, $\delta_{c m d}$ is the commanded fin deflection, $w_{n}$ is the natural frequency and $\zeta$ is the damping coefficient. The actuator model is selected with $w_{n}=60 \pi(\mathrm{rad} / \mathrm{s})$ and $\zeta=0.707$.

\section{Integrated Autopilot Design}

As discussed in the previous section, it is general to design the missile autopilot in roll, pitch and yaw channel, respectively. Since this autopilot design method does not consider the 
cross coupling dynamic properties, the effects of cross coupling such as the induced roll moment could not be handled. In this paper, new autopilot design structures with cross-feed loops are proposed to compensate the effects of cross coupling. Since the roll-pitch-yaw integrated system is a MIMO system, it is hard to find the combination of gains to meet the required control performance with the classical control design approach. In this research, the CEALM algorithm, one of the parameter optimization techniques, is employed to efficiently handle the gains of the proposed integrated autopilot. The advantage of CEALM is that it does not require the gradient information of the cost and constraint functions and is very robust to the initial guess of the solution. Gain optimization is performed with the linear model obtained at the trim points.

\section{Autopilot Structure}

Structure 1. The first proposed structure for the integrated autopilot is shown in Fig. 1. The structure 1 is composed of ten autopilot gains: three pitch channel gains, $\left(K_{p 1}, K_{p 2}, K_{p 3}\right)$, three yaw channel gains, $\left(K_{y 1}, K_{y 2}, K_{y 3}\right)$, two roll channel gains, $\left(K_{r 1}, K_{r 2}\right)$ and two cross-feed gains, $\left(K_{Z R P}, K_{Y R I}\right)$. In this structure, control commands of the pitch and yaw channels are cross-feeded to the roll channel in order to compensate the induced roll moment caused by the pitch and yaw accelerations.

Structure 2. The second proposed structure for the integrated autopilot is shown in Fig. 2. The structure 2 is composed of the nine autopilot gains: three pitch channel gains, $\left(K_{p 1}, K_{p 2}, K_{p 3}\right)$, three yaw channel gains, $\left(K_{y 1}, K_{y 2}, K_{y 3}\right)$, two roll channel gains, $\left(K_{r 1}, K_{r 2}\right)$ and one cross-feed gain, $K_{Z Y R}$.

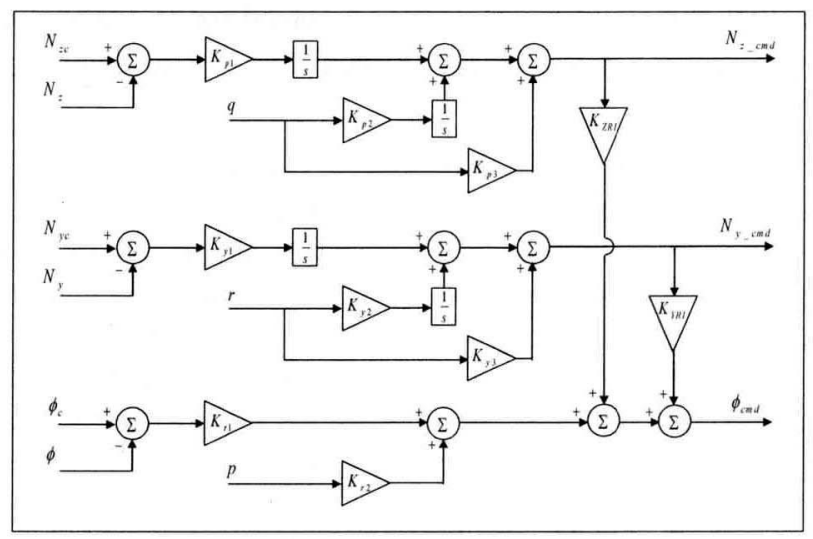

Fig. 1. Integrated Autopilot Structure 1

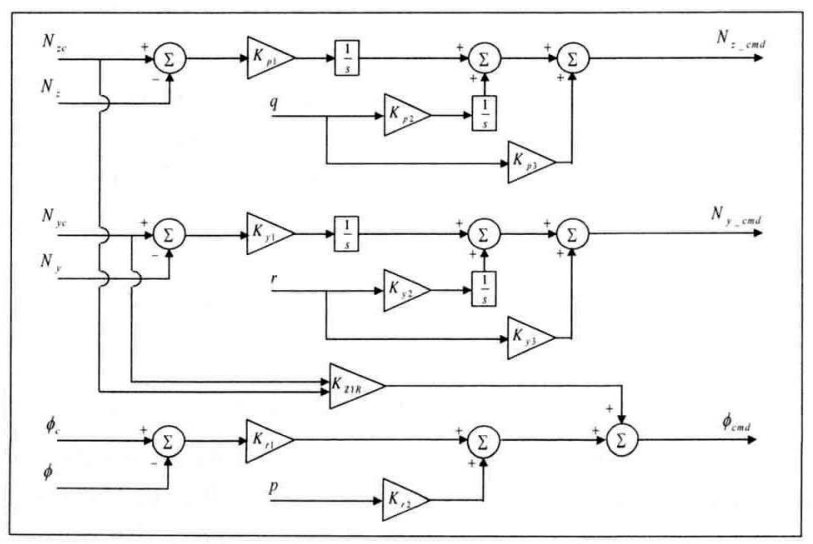

Fig. 2. Integrated Autopilot Structure 2 
If the velocity of the missile is constant, the induced roll moment is determined by the total angle of attack and the bank angle. Particularly, the induced roll moment can be illustrated as the sinusoidal function of the bank angle. Also, in the case of the cruciform missile, the induced roll moment has maximum value when the bank angle is 22.5 degrees. Thus the second structure of the integrated autopilot is proposed to compensate the induced roll moment by predicting the bank angle from the acceleration commands. The gain of cross-feed from the pitch and yaw channels to the roll channel is defined as follows:

$$
K_{Z Y R}=K \sin \left(4 \phi_{\text {wind }}\right)
$$

where, $\phi_{\text {wind }}$ is the bank angle caused and predicted by the acceleration commands and is calculated as follows.

$$
\phi_{\text {wind }}=\tan ^{-1}\left(\frac{N_{y c}}{N_{z c}}\right)
$$

\section{Cost Function}

Solutions of the optimization problem depend on cost functions and constraints. In an autopilot design problem using CEALM algorithm, the cost functions and constraints are chosen by considering the desired performance and requirements. To define the cost function, we formulate the integrated autopilot design problem as a parameter optimization problem with two candidate cost functions. This optimization problem is solved for the linear model linearized at the trim point with $10 \cos \left(45^{\circ}\right) \mathrm{g}$ normal acceleration, $10 \sin \left(45^{\circ}\right) \mathrm{g}$ yaw acceleration and 0 degree roll angle.

Method 1. The cost function of the method 1 is considered to minimize the error between the reference commands and the autopilot responses. The error terms are multiplied by time to minimize the steady state errors. The reference commands should be determined by reflecting the desired system performances. If the reference command is fast, large autopilot gains that may cause the saturation of fin deflections are obtained. In the method 1, the natural frequency and damping ratio of the reference command are $7 \mathrm{~Hz}$ and 0.707 , respectively. As an inequality constraint, we consider that overshoot for the acceleration command tracking should be less than $15 \%$. The cost function of the method 1 is given by Eq. (5).

$$
J=\int_{t_{0}}^{t_{f}}\left[\left(N_{z c}-N_{z}\right)^{2}+\left(N_{y c}-N_{y}\right)^{2}+\left(\phi_{c}-\phi\right)^{2}\right] \times t d t
$$

Method 2. The cost function of the method 2 is given by Eq. (6). In this method, the reference command is set as step functions. This cost function is considered to minimize the rise time and errors between the reference command and the accelerations after the rise time. The error of between reference command and roll angle is minimized from the initial time to the final time. The cost function of the method 2 does not need to consider reference command unlike in the method 1 .

$$
J=t_{r}+\int_{t_{r}}^{t_{f}}\left[\left(N_{z c}-N_{z}\right)^{2}+\left(N_{y c}-N_{y}\right)^{2}\right] \times t d t+\int_{t_{0}}^{t_{f}}\left(\phi_{c}-\phi\right)^{2} \times t d t
$$

The integrated autopilot structure 1 is adopted for the comparison of the cost functions. The linear model computed at the trim point with $10 \cos \left(45^{\circ}\right) \mathrm{g}$ normal acceleration, $10 \sin \left(45^{\circ}\right) \mathrm{g}$ yaw acceleration and 0 degree roll angle is used. Optimization results on the method 1 and 2 using the CEALM algorithm are summarized in Table 1 . Figs 3 and 4 show the numerical simulation results to verify the optimized control gains on the method 1 and 2 , respectively. 
Table 1. Optimized control gains of the structure 1 using the method $1 \& 2$

\begin{tabular}{|c|c|c|c|c|c|c|c|c|c|c|c|}
\hline & Cost & Kp1 & Kp2 & Kp3 & Ky1 & Ky2 & Ky3 & Kr1 & Kr2 & K_ZRI & K_YRI \\
\hline Method 1 & 1.8723 & -1.8513 & 1.9869 & 0.4097 & 1.2849 & -0.9303 & -0.4114 & 1.4110 & -0.0201 & 1.5088 & 1.4627 \\
\hline Method 2 & 1.1296 & -0.0177 & 0.0029 & 0.0401 & 0.0173 & -0.0090 & -0.0384 & 0.0746 & -0.0168 & -0.0969 & -0.0945 \\
\hline
\end{tabular}
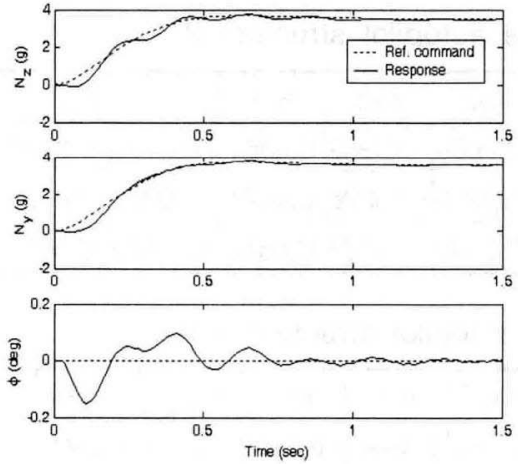
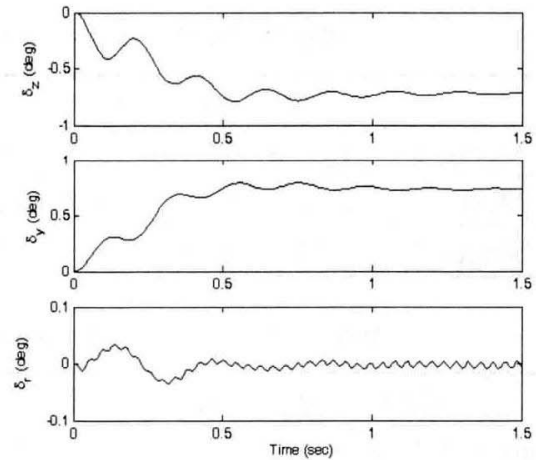

Fig. 3. Optimization result on the method 1
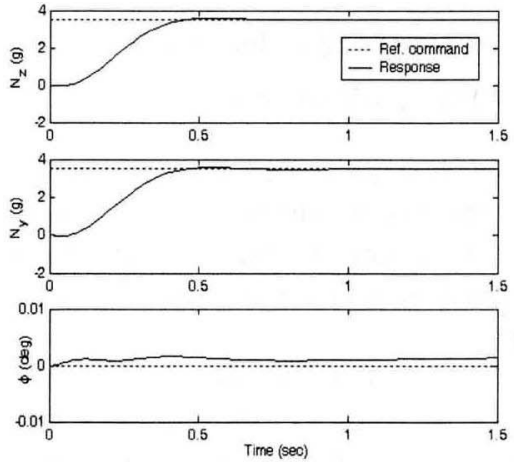
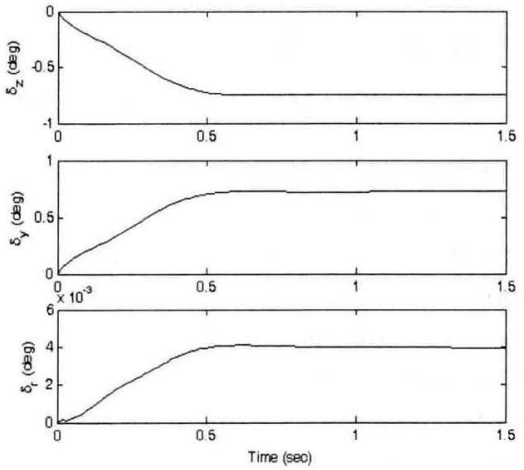

Fig. 4. Optimization result on the method 2

The optimized values of gains on the method 1 are larger than that on the method 2. From the results shown in Fig. 3, it is observed that the system responses oscillate because of the large value of autopilot gains. Also, since large gains may cause saturations of actuator, the method 1 is not suitable for the integrated autopilot design.

Fig. 4 shows that the system responses in the case of the method 2 track the reference command smoothly without oscillation. Not only the values of gain set on the method 2 are smaller than that on the method 1 , but also it has good performance with the low cost. Thus the cost function of the method 2 is adequate for the integrated autopilot gain optimization.

\section{Performance Analysis}

In this section, we analyze and compare the performance of the proposed integrated autopilot structures. In each case, we optimize the autopilot gains to minimize the cost function of the method 2. For performance analysis, let us consider three linear models which have the following trim conditions. 
Table 2. Optimization results using the autopilot structure 1

\begin{tabular}{|c|c|c|c|c|c|c|c|c|c|c|c|}
\hline & Cost & Kp1 & Kp2 & Kp3 & Ky1 & Ky2 & Ky3 & Kr1 & Kr2 & K_ZRI & K_YRI \\
\hline Model 1 & 1.7626 & -0.0270 & 0.0084 & 0.0644 & 0.0185 & -0.0061 & -0.0384 & 0.4810 & -0.0138 & -0.4859 & 0.4843 \\
\hline Model 2 & 1.1645 & -0.0115 & 0.0002 & 0.0401 & 0.0481 & -0.0203 & -0.0222 & 0.4276 & -0.0363 & -0.2348 & 0.2503 \\
\hline Model 3 & 1.1296 & -0.0177 & 0.0029 & 0.0401 & 0.0173 & -0.0090 & -0.0384 & 0.0746 & -0.0168 & -0.0969 & -0.0945 \\
\hline
\end{tabular}

Table 3. Optimization results using the autopilot structure 2

\begin{tabular}{|c|c|c|c|c|c|c|c|c|c|c|}
\hline & Cost & Kp1 & Kp2 & Kp3 & Ky1 & Ky2 & Ky3 & Kr1 & Kr2 & K_ZYR \\
\hline Model 1 & 10.558 & -0.0310 & 0.0021 & 0.0535 & 0.0284 & -0.0669 & -0.0352 & 0.4861 & -0.0190 & 0.0187 \\
\hline Model 2 & 2.1291 & -0.0139 & 0.0038 & 0.0646 & 0.0724 & -0.0260 & -0.0296 & 0.4871 & -0.0079 & 0.0095 \\
\hline Model 3 & 0.8705 & -0.0222 & 0.0053 & 0.0613 & 0.0207 & -0.0049 & -0.0123 & 0.4872 & -0.0179 & 0.0603 \\
\hline
\end{tabular}

Table 4. Optimized control gains of the autopilot structure $1 \& 2$

\begin{tabular}{|l|c|c|c|c|c|c|c|c|c|c|c|c|}
\hline & Cost & $\mathrm{Kp} 1$ & $\mathrm{Kp} 2$ & $\mathrm{Kp} 3$ & $\mathrm{Ky} 1$ & $\mathrm{Ky2}$ & $\mathrm{Ky3}$ & $\mathrm{Kr1}$ & $\mathrm{Kr2}$ & $\mathrm{K} \_$ZRI & K_YRI & K_ZYR \\
\hline Structure 1 & 176.18 & -0.0171 & 0.0011 & 0.0931 & 0.0368 & -0.0982 & -0.0651 & 0.4986 & -0.0287 & -0.3709 & 0.2241 & - \\
\hline Structure 2 & 85.42 & -0.0220 & 0.0051 & 0.0960 & 0.0385 & -0.0839 & -0.0456 & 0.4878 & -0.0101 & - & - & 0.0141 \\
\hline
\end{tabular}

Model 1: Mach 2.0, $N_{z}=10 \mathrm{~g}, N_{y}=0, \phi=0^{\circ}$

Model 2 : Mach 2.0, $N_{z}=10 \cos \left(22.5^{\circ}\right) \mathrm{g}, N_{y}=10 \sin \left(22.5^{\circ}\right) \mathrm{g}, \phi=0^{\circ}$

Model $3:$ Mach 2.0, $N_{z}=10 \cos \left(45^{\circ}\right) g, N_{y}=10 \sin \left(45^{\circ}\right) g, \phi=0^{\circ}$

These trim conditions represent the three different bank angles with $10 \mathrm{~g}$ total acceleration command and mach 2.0. The induced roll moment, one of the major effects of the cross coupling can be modeled as a sinusoidal function of the bank angle. Therefore we can compare the induced roll moment compensation performances of the two proposed autopilot structures by performing the integrated autopilot design on the three linear models.

The optimization results are summarized in Table 2 and 3 . In the cases of the model 1 and 3 , the cross-feed gain defined by the estimated bank angle becomes to zero. In these cases, the cross-feed loop of the autopilot structure 2 could not compensate induced roll momentums. Therefore, in the case of the model 1 , the optimization result of the autopilot structure 1 has better performance than that of the autopilot structure 2 .

On the other hand, in the case of the model 3, the induced roll momentum is negligible. Because not only the bank angle is 45 degree, but also the angle of attack and the sideslip angle are the same values. In this case, the autopilot structure 2 not using the cross-feed loop provides better performance than that of the autopilot structure 1 using the cross-feed loops.

The aforementioned trim conditions have the same total angle of attack, 8.198 degree. On the one total angle of attack, if the bank angle is not measurable, model variations induced by the bank angles could be considered as model uncertainty. To handle the uncertainty, robust control techniques such as $H_{\infty}$ and $\mu$-synthesis could be used. In this paper, we find the optimal gain set of the proposed autopilot structures to handle model uncertainty. In order to find the gain set to cover the three models, we define a cost function as follows:

$$
J=J_{1}+J_{2}+J_{3}
$$

where, $J_{1}, J_{2}$, and $J_{3}$ denote cost functions calculated from the model 1,2 , and 3 , respectively.

The optimization results using the structure 1 and 2 are summarized in Table 4. From the results, it is observed that the structure 2 provides better performance than the structure 1 . 
Since the autopilot structure 2 uses the cross-feed gain determined by the estimated bank angle, it is more robust than the autopilot structure 1 which has the fixed gain without reference to any bank angle. Fig. 5 and Fig. 6 show the simulation results on each model using the optimized gains of the structure 1 and the structure 2, respectively. Fig. 7 $\sim 9$ represent the nonlinear simulation results with the initial conditions of model 1,2, and 3, respectively. From the results, the nonlinear missile model is successfully controlled by the autopilot structure 2 with the optimized gains.
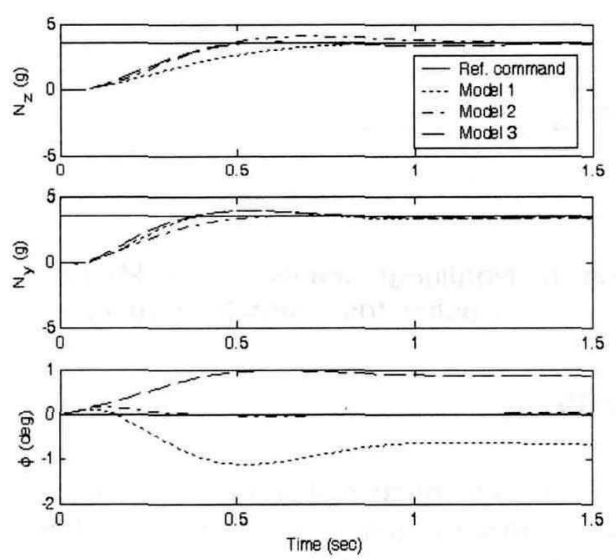
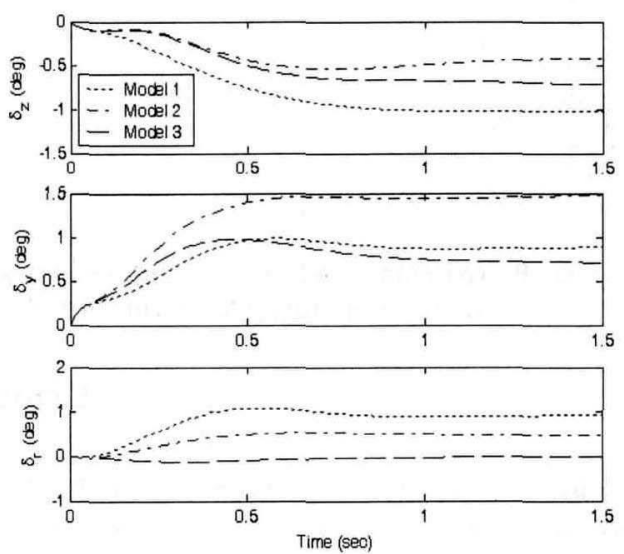

Fig. 5. Optimization result using the autopilot structure 1
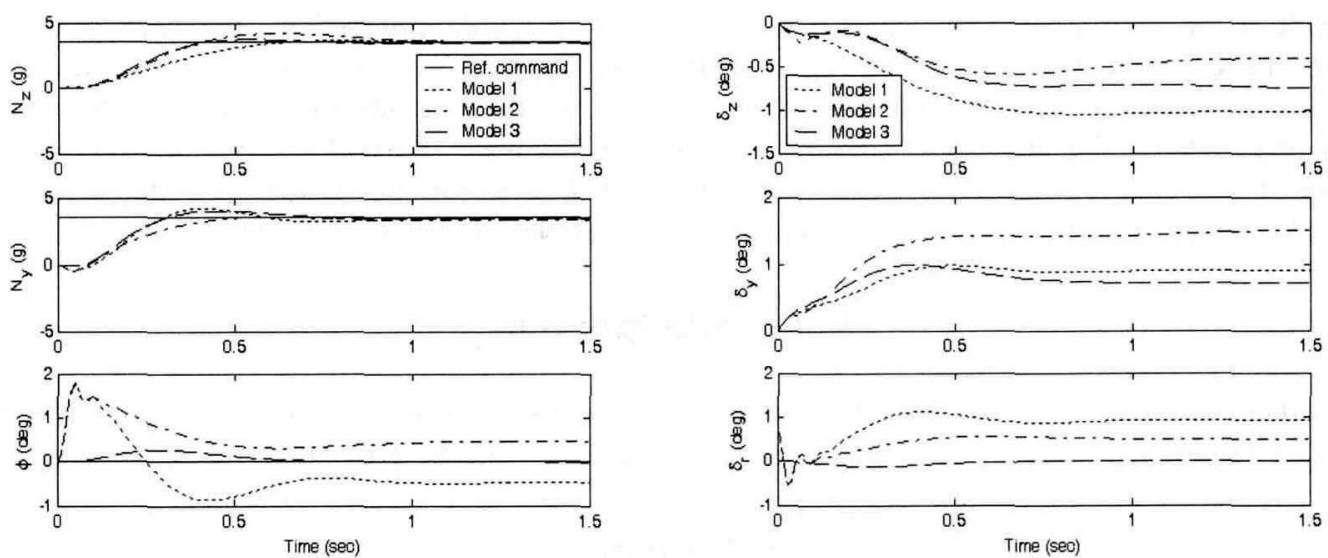

Fig. 6. Optimization result using the autopilot structure 2

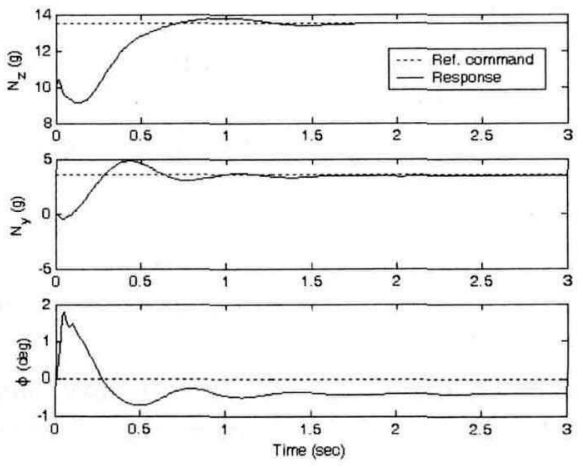

Fig. 7. Nonlinear simulation on Model 1 using the autopilot structure 2 

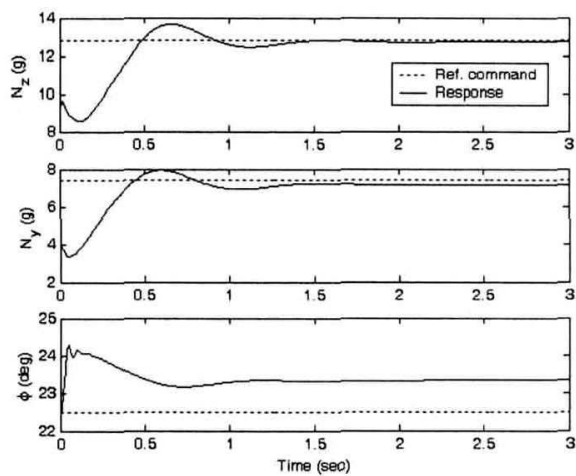

Fig. 8. Nonlinear simulation on Model 2 using the autopilot structure 2
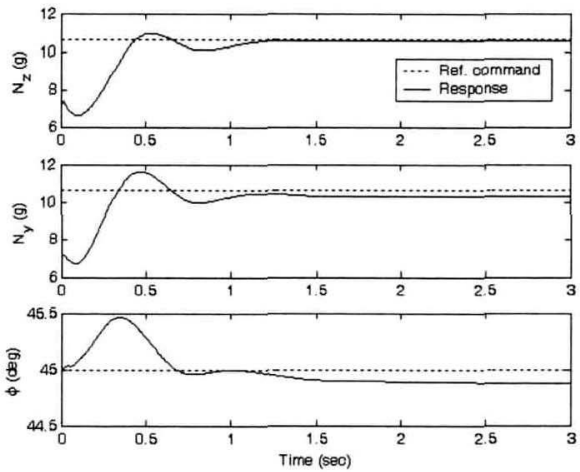

Fig. 9. Nonlinear simulation on Model 3 using the autopilot structure 2

\section{Conclusions}

In this paper we have proposed the two roll-pitch-yaw integrated autopilot structures to compensate cross coupling effects. One structure compensates the roll angle error caused by roll and pitch acceleration by cross-feed control commands of the pitch and yaw channels. The other structure compensates the roll angle error by cross-feed the bank angle predicted from the acceleration commands. In these autopilot design approaches, since the gains of the autopilot are difficult to be chosen systematically and the system is highly affected by the combination of gains, the CEALM algorithm is applied to determine the gains of the integrated autopilot. The simulation results of the two proposed autopilot structures demonstrate the tracking performances with existence of the induced roll moment. The findings from the performance analysis on the proposed autopilot structures suggest that the second structure is suitable for the integrated autopilot design to satisfy multiple models linearized at the different bank angles.

\section{Acknowledgement}

This work is supported by Agency for Defense Development, Daejeon, Korea, under the contract "Integrated Roll-Pitch-Yaw Autopilot Design for Missile with High Angle-Of-Attack."

\section{References}

1. W. R. Chadwick, "Flight Dynamics of a bomb with cruciform Tail", Journal of Spacecraft, Vol.4, No. 6, pp. 768-773, June, 1967.

2. W. R. Chadwick, P. Zarchan, "Interception of Spiraling Ballistic Missiles", Proceeding of the American Control Conference, Seattle, Washington, June, 1996. 1992.

3. B. L. Stevens and F. L. Lewis, Aircraft control and simulation, John Wiley \& Sons, INC,

4. H. Ryu and M. J. Tahk, "Automated Aircraft Flight Control Design Parameter Optimization Technique", KSAS Journal, pp. 111-119, 1998.

5. C. S. Park, J. Hur, and M. J. Tahk, "Aircraft Control System Design Using Evolutionary Algorithm", KSAS Journal, pp. 104-114,1999.

6. Min-Jea Tahk and Byung-Chan Sun, "Co-evolutionary augmented Lagrangian method for constrained optimization", IEEE Trans. on Evolutionary Computation, Vol. 4, No. 2, pp. 114-124, July, 2000. 